\title{
Scrutiny of Methods for Image Detection and Recognition of Different Species of Animals
}

\author{
Elham Mohammed Thabit A. ALSADI, Nidhal K. El Abbadi
}

\begin{abstract}
Animal detection-based study is useful in many real-life applications. Techniques involved in animal detection are useful in observing the locomotive behavior of the engaged animal and in result it prevent harmful interruption of animals in residential areas. There are some branches of research in animal detection. Some of these branches will therefore be discussed in this journal. Humans have developed many algorithms and techniques to gain a better understanding of animal behaviour. Therefore, for early preventive measures, these technologies can also serve as a warning system for humans from encroachment of dangerous wild animals. Such tasks can be reduced to three main branches, namely animal detection, tracking and recognition. Through these papers, new approaches for study and a variety of technologies/algorithms implemented in the past are identified and appropriate ways for solving the research gaps are suggested to fill the gap.
\end{abstract}

Keywords: Deep Learning, Deep Neural Networks, Artificial Intelligence, Camera-Trap Images, Animal Detection \& Recognition.

\section{INTRODUCTION}

Animal detection is a major and changing field due to a large number of real-life applications. Showing animal welfare on roads or in residential areas will use different methods of detection for the animal and warning systems. Requirements that are very relevant in real life are prevention of animal vehicle collision on highways, prevention of dangerous animal invasion in residential areas, awareness of targeted animal locomotive behaviour, etc. All of these technologies can be reduced to three fields, namely animal detection, tracking and identification. The very first area of animal detection was applied to different real-life applications areas. As an example, hundreds of camelvehicle accidents have been recorded each year resulting in numerous deaths and property damages involving millions of Saudi Riyals. Using global positioning system technology, a deployable and intelligent Camel Vehicle Accident Avoidance System (CVAAS) was designed to address this problem. Researchers in developed a Light Detection and Range Data Algorithm (LIDAR) to enable fishermen to find the right place for deep-sea fish [21]. Scientists use micro-Doppler signals to ensure human health and security by detecting potential dangerous animal intrusions into the residential area.

The second branch focuses on monitoring animal locomotives and their contact with the animal tracking environment. One application is the development of new animal animal trace system geology applications, monitoring and theft control and safety in zoos[36] for sensor technology, radio frequency identification (RFID) and global positioning system (GPS).

Revised Manuscript Received on November 11, 2019.

* Correspondence Author

Elham Mohammed Thabit A. ALSADI, College of Information Technology, University of Babylon, Iraq

Nidhal K. El Abbadi, Education College, University of Kufa, Iraq
It helps people to have a better understanding of living creatures on earth by monitoring animal movements, particularly how the animal interacts with its environment. The third element, the animal identification, is used to classify the animal that has been identified. It was used for domestic animals in the health monitoring program [22, 35]. Management of animal care becomes an important issue because animals directly affect the psychological and physical health of humans.

Although there are many remedies, animal abuse and animal health threats are on the rise. In order to better control animals in the retrieval of complex data, location tracking and RFID-based mobile monitoring system (RFID-MMS) are designed to assist users over a wireless network [23]. Animal identification has made it easier for people to track and control their pets. This paper will pay more attention to animal detection methods and will study them. The analysis also prohibited the use of digital images or digital video techniques. In the next chapter, the review will be issued.

\subsection{Animal Detection Methods in Field Of Image} Processing

The field of Animal detection is a major area in study of various applications. Techniques of animal detection are useful in studying targeted animal's locomotive behavior and also to prevent dangerous animal invasion in residential areas. Early research on the identification of animals is to determine how easily human eyes can detect the presence of animals in the natural world. Animal detection by human eyes was seen from the computational point of view as the most reliable method of detection [24]. This is due to the ambiguity of the object form in natural objects. It is found in that a human observer can determine if a quickly flashed animal scene includes an object as fast as $150 \mathrm{~ms}$. In the results of median reaction time suggest a speed accuracy of 92 percent for $390 \mathrm{~ms}$ reaction time and increase to 97 percent for $570 \mathrm{~ms}$ correctness. Although human detection is successful and achieves a satisfactory level, human eyes can quickly become tired, resulting in reduced efficacy. In fact, human eyes are unable to function 24 hours a day to identify animals. The use of computer vision in image processing [18] for animal identification will counter these flaws.

The main task is ecology in its natural environment, that follows wild animals. Speedy population growth and endless growth were over-exploitation of natural resources and result in rapid, novel and significant changes to the country's ecosystems. The through amount of land area, species change patterns, environment and actions have been changed by human action. More importantly, various species of animals are being thought to be extinct on earth and many are being introduced in new fields that can damage both natural and human systems. Several contemporary techniques, including radio tracking, wireless sensors and camera sensitive trap detection, have been produced for wild animally monitoring [ 25 ]. Motion-led

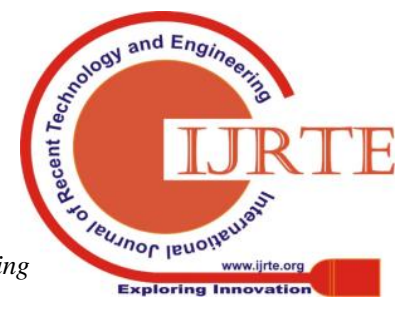


remote cameras or "camera traps," that have novel features equipped for wide trade accessibility and ease of deployment and use, become a more and more common tool for wildlife control. A standard coated camera model can collect time, temperature and moon phase information, incorporated into the image data, to capture strongdetermination images both day and night [37 ].

In addition, generous and versatile camera setup allows for secret and consistent follow-up of animals. A camera can take thousands of images and provide a lot of information when completely loaded. These standards make camera traps a powerful tool for geologists to document all wildlife aspects [27 ]. These standards are a powerful tool to environmentalists as camera traps can document all wildlife aspects. Camera trapping is being carried out quickly in the field of wildlife monitoring with digital technological improvements, which produce more contemporary camera traps with system part automation, but reduce costs for procurement [26,38]. Although the human visual system can quickly and easily process images, manually manipulating such a large amount of images is still very costly. Although the human visual system can easily and quickly process a large number of pictures manually, it is very wasteful. Furthermore, the large collections of images and shortcomings in image quality, including experts, are an important factor in the speed and precise human evaluation. Some photos in Snapshot Serengeti are classified as ' unidentifiable ' in particular, more than 9600 images were marked as ' anyway ' or ' video problems, ' and thousands were marked inconsistently in the data set of Wildlife Spotter and Victoria South Central. In the past years, various governments and organizations have invested additional funding in the protection of the habitats of various animal species which are at risk in their natural environment, particularly by means of the construction of new facilities [27 ]. When new infrastructure is growing, animal migration corridors are challenging. This is the main reason for implementing an embedded automatic system with artificial intelligence components to monitor animal activity and to provide data about animal migration in a specific area. This program will replace standard methods that could not span a period of time (direct observation, field routes, droppings, etc). When planning and financing a new highway system, information on wildlife migration opportunities can be used to reliably model foraging corridors. The animal detection system in the desert of Mojave was used to defend endangered and endangered species from animals in wildlife. The expressions of the eyes of internal emotional states play an important role in human and other primates. Additionally, continuous knowledge of primate facial expressions may increase mental health and behavior. Facial expressions are standard for human beings and share most common characteristics in cultures Technologies for the detection of human feelings are more and more automatic and reliable due to increased computation capacity and space availability. Non-human primates remain unable, despite this development, to identify facial expressions and evaluate emotional states by using automatic means which prevent the development of animal models in mental health researchNo instruments are available for detecting facial expressions automatically and assessing emotional states in semi-homan primates, in spite of such innovations, which impede animal models for studies on psychological health. The AFER decodes a range of predetermined people's emotions, along with happiness, tristy, incredulity, disgust and surprise.

Despite these developments, automatic instruments to detect facial expressions and evaluate emotional outcomes for nonhuman primates have not been available for the tracking of animal models in mental health research. Given these advances, for non-human primates, manual equipment exist for identifying face expressions and assessing emotional state, impeding animal models of mental health studies. AFER decodes a range of predetermined stimulations such as happiness, liking, sorrow, anger, surprise, fear, and surprise. AFER decodes pre-determined emotions like joy, sorrow, rage, disgust, confusion or anxiety for people. AFER schemes have variability among subjects and the objective challenge for some adaptive states such as having to suffer from pain or depression [28] in finding correct grounds for truth. AFER algorithms in humans are primarily based on models or model-free statistics. Model-based techniques naturally assume a pre-determined prototype of expressions and are directly linked by Action Units (AU) to decoding muscle activity blocks of facial expressions Each $\mathrm{AU}$ has its own number of muscle movement and facial appearance qualities.

? Any more organized decision-making method, including fundamental affective recognition, could be used by Aus. The Facial Action Coding System (FACS) is configured to objectively and deeply decode human facial expression. Model-free techniques are based on the use of greatly training data sets, such as profound neural network learning with pre-labeled voice inflections, with statistical machine learning instruments. The detection and recognition of animals is usually a challenging task and no special technique offers an efficient and robust solution for all situations. The algorithms for animal detection are commonly done as a binary classification task in animal detection[30]. That is, the input image is divided into blocks and translated into an element in every block. A certain class is used to train features of the animal relating to a given class Model-free methods are based on the program, such as in-depth learning based on convolution neural network, of statistical tools with massive training sets with re-marked facial expressions. The detection / recognition of objects stays a difficult challenge and there is no specific approach that can address all problems in a stable and efficient way. Broadly speaking, animal detection algorithms is used as a binary classification task [17] for animal detection. Then the classifier will decide if the sample is the animal, when a new input image is given. For the following basic applications the animal recognition system classification can be seen in Figure 1: 


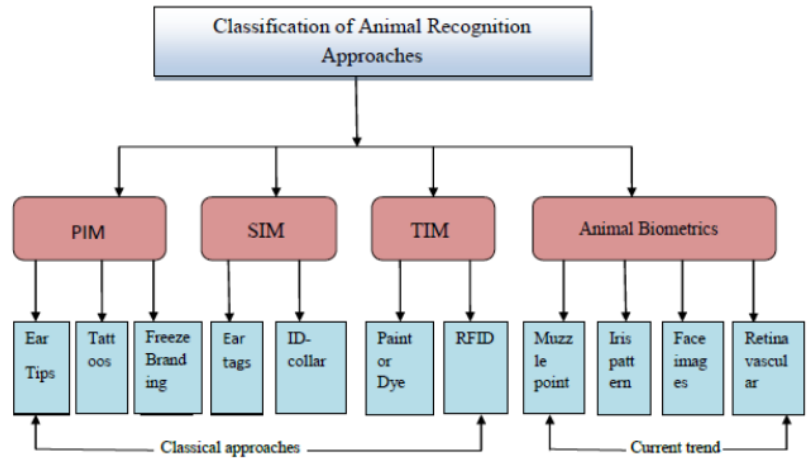

Figure 1 Classification of animal recognition approaches

[14].

? Compares the animal and confirms or continues to refuse the identity of the discovered animal (the one - to-one matching). Even though verification and identification often share the same classification algorithms, the two modes are intended for specific applications[17 ].

[? Identification-compares the picture of the animal with all other animals in the database and gives the matches a categorized list (one to $\mathrm{n}$ matching).

[? To better comprehend the task and issues of animal identification and recognition, the following factors have to be considered because the outcomes of animal detection and recognition system may be greatly impaired:

? Illumination and other image acquisition conditions -variables such as light differences in source distribution and intensity or camera characteristics such as sensor reaction and lenses can be influenced by the animal picture input.

? Occlusions-Animal images can partially be overlooked by other objects and other animals

Object detection (OD) is a technologically difficult and practically helpful computer vision issue and has seen important progress in recent years [1]. In computer vision (CV) and picture processing, detection and classification of objects are significant tasks. Classification of objects could be considered a normal problem of recognition of patterns. Unlike people who can easily recognize any object in the actual globe, it is a hard job to computerize object recognition in picture. The Detection and classification of animals is a challenge for the scientist owing to numerous problems that decrease performance and effectiveness. Effortlessly and instantly, humans conduct object recognition. Algorithmic description of this assignment was very hard to implement on computers.

The basic model for object detection is shown in Figure 2. Basically an OD scheme can be readily defined by seeing Figure 1, showing the fundamental phases engaged in the object detection method. In the case of videos, the basic input to the OD system may be an image or scene. The fundamental purpose of this scheme is to identify objects (animals) current in the picture or scene, or merely to categorize the different objects (animals) into the corresponding object classes. The issue of object detection can be described. Due to an image comprising one or more interesting objects and a set of labels corresponding to a set of models known to the system, the system is intended to assign accurate labels to the regions of the image. The problem of object detection cannot be solved until the image is segmented and segmentation process cannot be implemented without at least partial detection. The word detection was used to refer to numerous distinct visual capabilities which include the identification, discrimination and categorization. In this paper we will discuss the various techniques and methods adapted by the researchers in past few years.

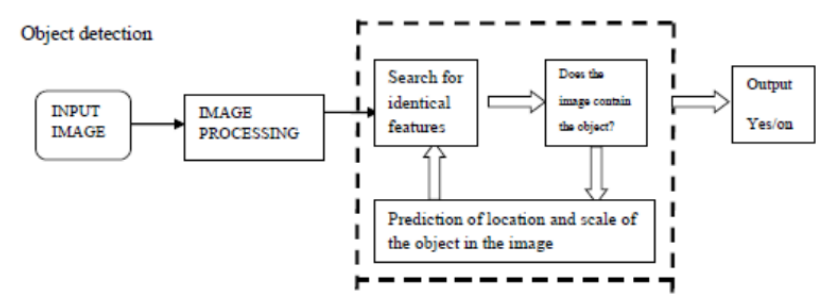

Figure 2. Basic Objects Detection Model [13]

\section{RELATED WORK}

In numerous applications research has been pulled out into animals in image processing Many human algorithms and techniques have been established to better understand evolutionary biology. In addition, these applications serve as an early warning mechanism for people against harmful intrusions to wild life[15].

Boon Tatt Koik et al. (2012) Survey proposed to detect, track and recognize animals in some of these branches. Research based on animal detection is helpful for many real life applications. Animal detection methods are useful in researching the targeted animal's locomotive behavior and also in preventing hazardous animal intrusion in residential areas. First is the issue of lighting, where a sudden shift of lighting impact can influence the efficiency of identifying the presence of animal intrusion mostly in indoor applications. In addition, the detection may also be affected by the luminance issue with day-to-night modifications in the natural environment at the outdoor monitoring scheme. Furthermore, moving background, such as such as wind leaves could be considered as a foreground image and the algorithms could erroneously interpret some inactive animal that remains static for a long time as a background image. Visual descriptors were chosen to develop object representation, and descriptors were classified using Support Vector Machine (SVM).

Ferryman, J., et al. (2013) addressed a technique of video surveillance that detects abandoned objects in surveillance scenes robustly and effectively. This technique used a fresh algorithm for threat assessment that incorporated ownership notion with automatic knowledge of social relationships to infer object abandonment. Implementation was accomplished by developing a logic-based Prolog-based inference engine. The efficiency of threat detection was performed by testing against a range of data sets describing realistic scenarios and showing a decrease in the amount of false alarms produced.

Slavomir Matuska et al. (2014) A new automatic animalrecognition system (ASFAR) and grade system were described in this paper. AFSAR collects data from its wild nature watch tools. System detection track animals and then send information to MCU. This information is reviewed by MCU and animal migration corridors are built in the given

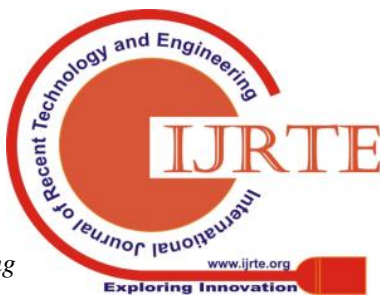


country. In MCU, SVM classifier BOW software was deployed in c. This technique of classification has been tested in our experiments. This technique of classification has been tested in our studies. The research demonstrates that an algorithm in terms of dealing with the SURF detector, SIFT descriptor, Flann Based matcher, 18,000 clustering descriptors and 20,000 learning descriptors achieved the highest grade score of 94 percent.

Slavomir Matuska et al. (2014) in this paper proposed a novel method based on hybrid descriptors is suggested for object recognition. This technique uses several methods (SIFT-transforming invariant-scale features, SURF-robust speed-up features) and consists of two components. Some pictures from the dataset demonstrate the applicability of the hybrid techniques submitted. Classes of data set include the wolf, fox, brown bear, wild boar and horse-drawn livestock in the Slovak nation. The technique shown can also be used in other fields of classifying images and extracting feature. The experimental findings indicate that local descriptor combinations have a positive impact on the detection of objects. It presents and tests two hybrid main pointsdetectors compared with other detectors. The BOW and SVM classificator combinations have been tested. Experiments have indicated that an algorithm based on SISURF, Opponent SIFT Descriptor, the Brute Force matcher and 15000 descriptors in a clustering system has attained a highest grade achievement rate of 86 percent. In addition, in four other cycles, success rates of over $80 \%$ were accomplished. The proposed SISURF hybrid key detector produced promising outcomes similar to other key point detectors. In addition, other conventional detectors were outperformed by SISURF.The proposed SISURF hybrid key point's detector promising results that are comparable to other key point detectors.

Peter Christianse et al. (2014) The automatic method for the detection and validation of animals using thermal cameras in the UAV company is introduced by On the basis of a complex limit, hot points are identified and the trademark extraction is carried out. The new technique of extraction of thermal signature described in this article is the extraction for each subject and a parameterisation based on DCT. A grading technique is presented with measurements from single and multiple frames. With a balanced classification precision of 93.5 percent in the altitude range of $3-10 \mathrm{~m}$ and 77.7 percent in the $10-20 \mathrm{~m}$ altitude, the combination of multi-frame measurements achieves the highest performance, thus showing a clear connection between investigator performance and altitude classification. The proposed system for detecting and classification is based on wildlife top-view images as seen by a UAV. In terms of performance of the whole algorithm the simulated and limited dataset is favorable. The identification and classification model proposed is based on highest level-of the-range images seen by a wildlife UAV. The automatic tracking and recognition of wildlife by UAV software is primarily part of ongoing wildlife-friendly agriculture study. In the paper, a concept is proposed for CNN (PCA, LDA, $\mathrm{SVM}$, and LBPH) in contrast to the well-known frame recognition, extraction and object classification. The suggested CNN was analyzed on the animal database created.

Zhang, T., et al. (2015) addressed the problem of current research in kangaroo such as population tracking and monitoring of activities. However, traditional manual observation, especially for wild kangaroos, is not as effective and expensive as automated systems. A significant step in experimentation with computer vision instruments is to help study kangaroos in the wild. A kangaroo picture dataset was developed to investigate feasibility from gathered information from various domestic parks throughout Queensland. A Multi-position strategy was studied, and a framework was suggested to obtain reasonable detection precision by using the state-of - the-art Deformable Part Model (DPM). Since DPM cannot detect kangaroos caused by various poses

Kumar, S., \& Singh, S. K. et al. (2016) Focused primarily on pet animal tracking in Smart City, a significant challenge for authorities. The required level of animal safety and management is not delivered for by classic processes of animal identification and tracking. In this report, it suggested the use of the key animal biometric identifier to track individual animal animals (dogs). The recognition technique was used to match and classify the extracted features of pet animals (dogs) with single-shoot impermissibility and distance metric approaches. A prototype was developed to evaluate the accuracy of the recognition system.

Tibor TRNOVSZKY et al. (2017) Proposed a device input for the Convolutionary Neural Network (CNN). This approach is contrasted with well-known image recognition approaches such as Primary Binary Pattern (LBPH) Histograms and System Valve (SVM) Linear Discriminate Analysis (LDA). The primary purpose is to equate the general recognition accuracy of PCA, LDA, LBPH and SVM with the existing CNN technique. For the experiments, the wild animals database is created. This database contains 500 various subjects ( 5 classes/100 images per class). The wild animal database is created for the experiments. There will be 500 subjects in this list ( 5 classes / 100 images per class). The overall performance was obtained by various training pictures and test images. The experimental results indicate that the approach described has a beneficial impact on animal recognition's overall performance and exceeds other techniques tested. The article presents an expected CNN (PCA, LDA, SVM and LBPH) compared against wellknown algorithms, for the identification, extraction and object classification of images. The proposed CNN was tested in the generated animal database. For different training images and test images the overall performance was obtained. Several learning objects and videos were used to generate the general results. The results show that the LBPH algorithm produces the best results for large running groups than PCA, LDA and SVM. The requested system achieves a precision of almost 82.5 percent for animal (cow) detection. The proposed system can be used with other current and active pedestrian and car detection technologies to prevent accidents and loss of human life on roads as a full solution (Package)[19].

Jaskó, G., et al. (2017) provided a system capable of identifying from traffic scenes various enormous wild animals. Visual information from a monocular color vision camera was acquired. The goal was to evaluate the image of the traffic scene, identify the areas of concern and classify them properly for the discovery of the animals that were on the highway and could trigger an accident. Using intensity, color and orientation features, a saliency map was generated 
from the image of the traffic scene. It was presumed that the outstanding areas of this map were areas of concern. A database of a big amount of pictures comprising multiple wild animals with four legs was collected. A database was compiled from a large number of images containing various four-legged wild animals. Relevant features were extracted from these and were utilized for training Support Vector Machine (SVM) classifiers. These classifiers provide an accuracy of above $90 \%$ and are used to predict whether or not the selected regions of interest contain animals.

Hung Nguyen et al. (2017) proposed and demonstrated the feasibility of constructing a scalable automated wildlife surveillance system with a deep learning strategy. In order to inform conservation and management decisions, efficient and reliable surveillance of wild animals in their natural habitats is essential. Use the Wildlife Spotter dataset in this paper, which includes a big amount of pictures taken by trap cameras in South Central Victoria, Australia. In recognizing pictures with animals, this model accomplished more than 96 percent and nearly 90 percent in identifying three most common animals (bird, rat, and bandicoot).

Rey N., et al. (2017) investigated a semi-automatic system capable of detecting large mammals in semi-arid Savanna was explored. It uses machine learning to rely on an animal detection scheme, trained with crowd-sourced annotations supplied by volunteers who manually interpreted color pictures with sub-decimeter resolution. A high recall rate has been accomplished for the scheme and a human operator can then with restricted effort remove false detections. The system offered excellent prospects for developing datadriven wildlife conservation management methods.

Tarrit, K., et al. (2018) addressed the significance of visual surveillance in public spaces and especially in train and subway platforms that are particularly vulnerable to many kinds of crime from small-scale theft to terrorist activity. If image quality cannot be enhanced by means of highresolution sensors, high-end lenses or IR illumination, the visual surveillance system may need to increase the image resolution capacity by software to provide accurate outputs such as disappearing points (VPs) in our case. In this work, we provided a three-stage technique for accurately detect the primary lines and vanishing points (VPs) in low-resolution images obtained in indoor and outdoor railway platform settings through visual surveillance systems. Initially, several frames were used by a multi-frame picture enhancer to increase the resolution capacity. Adaptive edge detection was then performed and a new line clustering algorithm was applied to determine the parameters of the lines that converge at VPs; this used the detected lines and heuristics statistics about the type of scene. Finally, gaps were calculated through a voting system to optimize detection in an effort to omit spurious lines. The proposed approach is very robust as it is not affected by ever-changing illumination and weather conditions of the scene, and it is resistant to vibrations.
Gyanendra K. Verma et al. (2018) focus on tracking and analyzing animal wildlife through the identification of natural scenes from camera trapping systems. The camera trap picture sequences consist of extremely encroached pictures which prevent animal detection leading to low detection rates and high false detection rates. We used a camera trap database that has animal proposals candidates with a multilevel graph cut in the spatiotemporal domain to deal with this issue. These proposals are used to produce a check stage which will determine whether a particular patch is animal or background. In this study we have taken animal movement and the spatial context into account in developing candidate animal areas, using the IEGC method for analyzing the camera-trap picture sequences. In addition, we have noted that the picture functions of the Deep Convolutionary Neural Network (DCNN) function well and enhance classification efficiency using separate classifiers. We have intended a solid and reliable camera-trap picture model that is extremely dynamic and cluttered.

Eric T. Psotaet al. (2019) introduced a fully convolution neural network used to identify the location and orientation of multiple group-housed pigs. The target output of the network is goal is to display an image space of each part of the pig along with a method for associating them to form complete instances. A new dataset with 2000 images and 24,842 pig cases is used to train the network. The dataset is divided into a training set and a testing set, and the testing set is subdivided into two sets: one with images depicting the same environments as the training set, and another with images of new environments not represented in the training set. This dataset design allows the robustness of detection algorithms to be tested against novel animal presentations and environments. The three key contributions of this work are:

(1) A fully convolution instance detection method,

(2) A public dataset for training and evaluation, and

(3) The design and/or training methodology should be revised to improve the robustness to environmental variability.

Regarding the third interpretation, it is not possible for spatial transformation to mimic changes in luminous conditions while significant increase has been applied to the input and output images during training. Therefore, a new set of non-uniform color-space transformations may provide a solution that improves the robustness of the trained network.

\subsection{Animal Detection Systems and Algorithms Comparison}

An in-depth literature analysis brought forward many innovative animal detection systems / algorithms. Although the number of publications on this particular topic is smaller than that of vehicle detection (another important factor of road safety), we found a variety of animal detection algorithms. The figure below illustrates different techniques used so far to identify animals. This involves the identification and tracking of endangered species. 


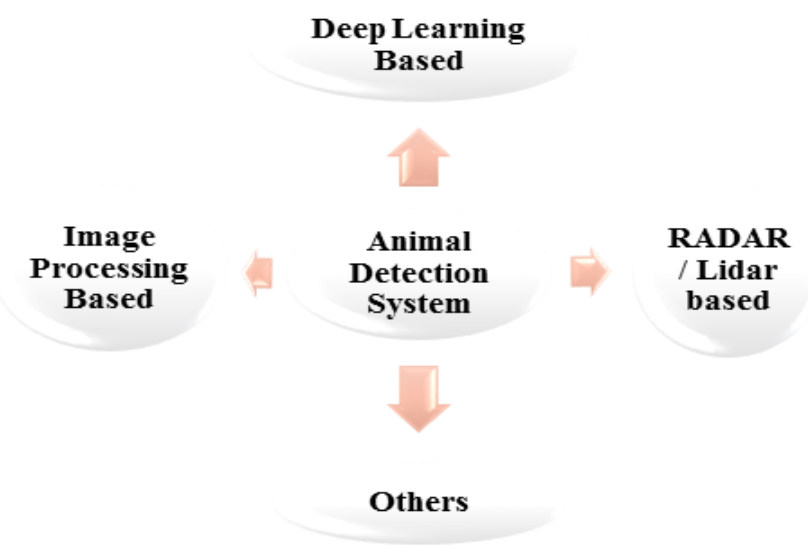

Figure 3 Different techniques used for detecting

Table 1 Comparison of different images and videos based object detection techniques

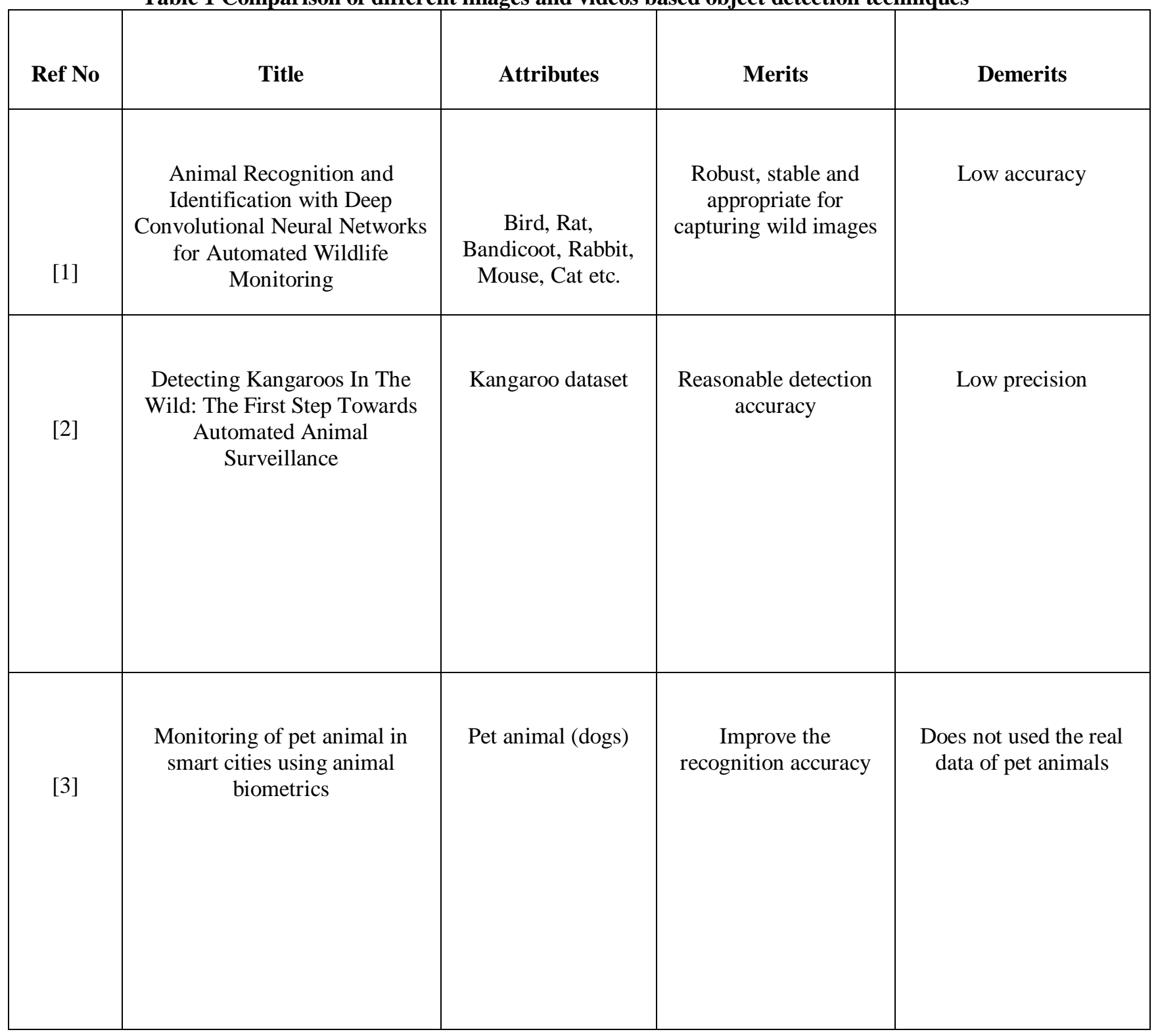




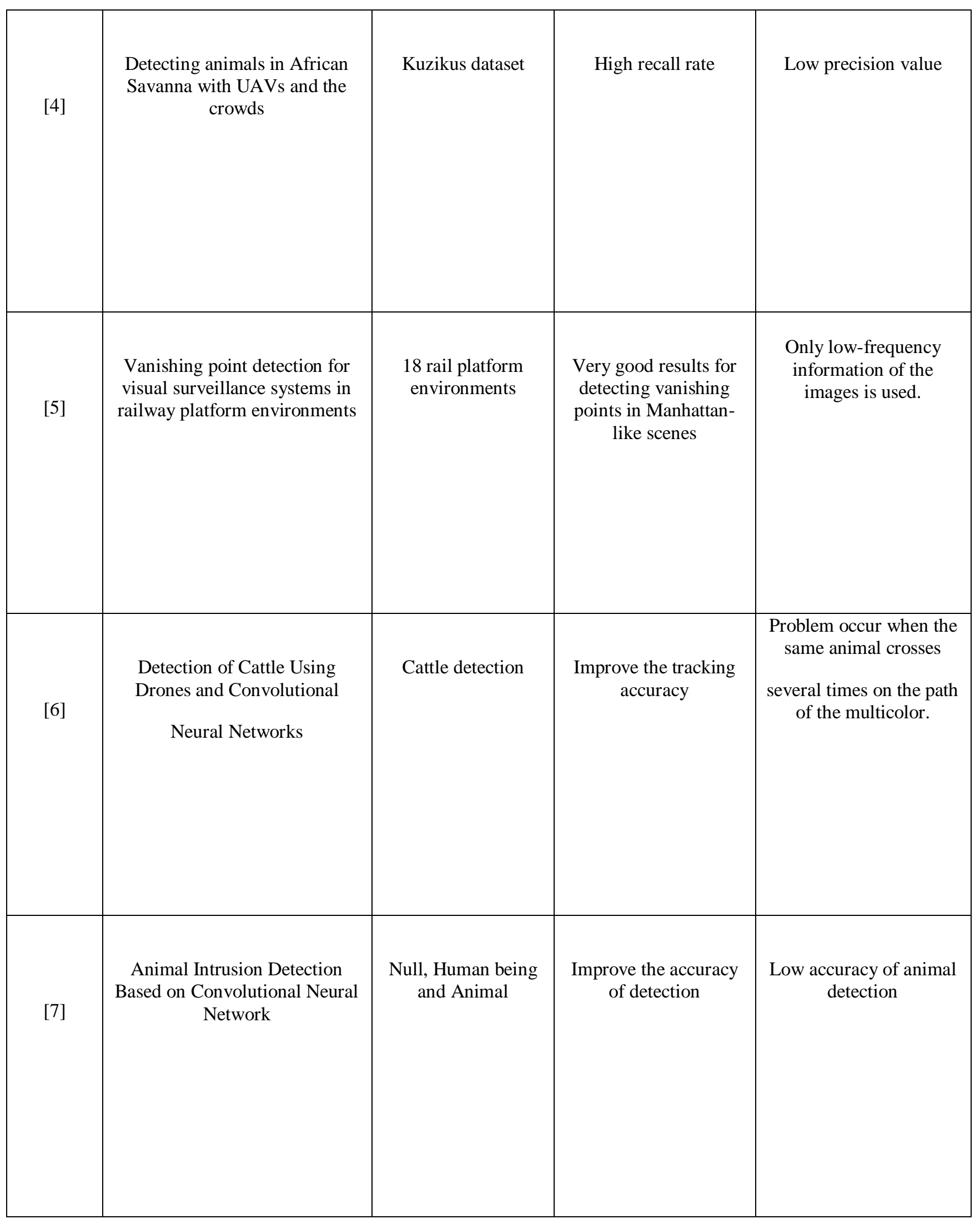




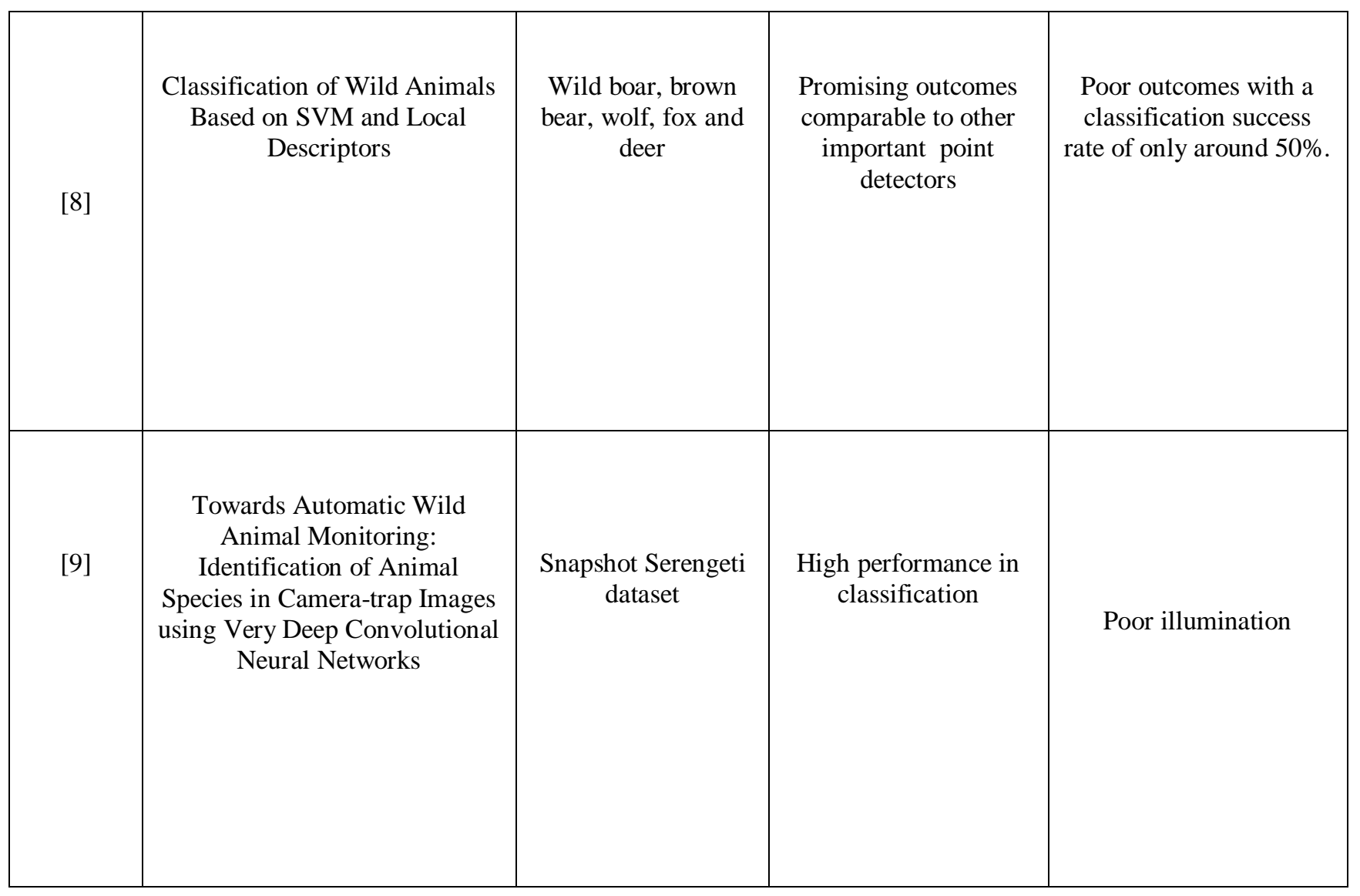

Table 2: Algorithms for detecting animals

\begin{tabular}{|c|c|c|}
\hline Reference & Algorithms and Techniques & Implementation \\
\hline$[10]$ & Foreground-background segmentation and object verification & $\begin{array}{c}\text { Detects animals in highly cluttered } \\
\text { videos }\end{array}$ \\
\hline$[11]$ & Fusion of deep learning and HOG features & $\begin{array}{c}\text { Robust algorithm Detects animals in } \\
\text { highly cluttered natural images }\end{array}$ \\
\hline$[12]$ & Deep CNN with machine learning & $\begin{array}{c}\text { Accuracy of 91\% and works for both } \\
\text { daytime and nighttime }\end{array}$ \\
\hline
\end{tabular}

Table 3: Performance comparison of above mentioned algorithms

\begin{tabular}{|c|c|l|c|c|}
\hline Study & Year & \multicolumn{1}{|c|}{ Method } & Precision & F-score \\
\hline Zhi Zhang et al. [10] & 2015 & $\begin{array}{l}\text { Ensemble graph cuts for object } \\
\text { classifier as foreground-background } \\
\text { segmentation followed by object } \\
\text { verification }\end{array}$ & 0.8293 & 0.8695 \\
\hline Zhi Zhang et al. [11] & 2016 & $\begin{array}{l}\text { Combination of deep learning and } \\
\text { HOG features }\end{array}$ & 0.8209 & 0.8398 \\
\hline $\begin{array}{c}\text { Animal Detection } \\
\text { with deep CNN [12] }\end{array}$ & 2017 & $\begin{array}{l}\text { Deep CNN features with machine } \\
\text { learning }\end{array}$ & 0.91625 & 0.9476 \\
\hline
\end{tabular}

\subsection{Research Gap}

The various practical solutions available for automatic detection and recognition of animal for highways are going on. These are the gaps we found during automatic animal detection:

[? Animals in distinct forms, dimensions, stances, colors, and conduct are not completely predictable in comparison with walking or other objects, which are the biggest problem.

[? Although human beings have a beautiful basic shape and size, animals do not share the average and standard.
? Animal detection is comparatively easier if the device is looking for an animal (say cat) to be detected. However, inherently acknowledging many animals needs the ability to distinguish between one animal and the other, although they may not be of the same sort. Such a issue for computers is very hard if they do not understand about animals' different opportunities.

? Given the complications apart from a moving or stationary animal, along with the interest involved in identifying the animal on the roads, there are other objects such as vehicles, pedestrians, 
shade of trees and other objects that make the animal very difficult to detect.

\section{CONCLUSION}

We reviewed the literature on the automatic detection and classification of animal image. We faced many problems during the detection and image classification of animal such as large variation in shape and color appearances, light/illumination conditions problem. In recent years, with the increasing importance of animal identification and tracking, animal recognition algorithms have received more and more attention, and the application of neural networks has opened up new and individuals. This paper uses this principle to use convolutional neural networks for animal identification studies. The experimental results show that the optimal feature extraction algorithm and optimization algorithm are obtained. It is hoped that later researchers will be able to conduct more in-depth research on this basis to further improve the performance of animal recognition algorithms based on neural networks for animal identification. Animal characteristics help to better describe and identify different species. However, there still exist research gaps for the further improvement and innovation in this area.

\section{FUTURE SCOPE}

Most animal detection systems are found to have their own advantages and disadvantages. Yet in a country like India, given the growing number of accidents with stray animals, there is no realistic implementation of such systems. Future research needs to expand in order to develop a device capable of detecting animals during the day, at night and also in fog. In foggy weather, none of the existing systems promised to detect animals. In fact, most AVCs (AnimalVehicle Collision) occur when dogs or cows strike the vehicle in urban environments, so we need to concentrate on identifying animals like dogs and cows instead of moose or giraffe. The effectiveness of a system depends on two factors: the reliability of detection and the signaling of the animal to the driver. In terms of detection accuracy, the technologies / algorithms studied above indicate that a rained DCNN performed quite well by giving approximately $91 \%$ precision, $91-93 \%$ true positives and $6-10 \%$ false positive rate[16]. On both day and night, it performed well. In contrast, RADAR is also capable of working in harsh weather. Those two can be further expanded to make it work on highways (background continues to change) and in real time. The most important fact to communicate with the driver is to tell well in advance so that the driver has ample time to take the action. All of these criteria have created a large area for further animal detection and alarm system work to be applied on a large scale.

\section{REFERENCES}

[1] H. Nguyen et al., "Animal Recognition and Identification with Deep Convolutional Neural Networks for Automated Wildlife Monitoring," 2017 IEEE International Conference on Data Science and Advanced Analytics (DSAA), Tokyo, 2017, pp. 40-49.

[2] Zhang, T., Wiliem, A., Hemsony, G., \& Lovell, B.C. (2015), Detecting kangaroos in the wild: the first step towards automated animal surveillance. 2015 IEEE International Conference on Acoustics, Speech and Signal Processing (ICASSP), 1961-1965.
[3] Kumar, S., \& Singh, S.K. (2016). Monitoring of pet animal in smart cities using animal biometrics. Future Generation Comp. Syst., 83, 553-563.

[4] Rey, Nicolas \& Volpi, Michele \& Joost, Stéphane \&Tuia, Devis. (2017). Detecting animals in African Savanna with UAVs and the crowds. Remote Sensing of Environment. 200. 341-351.

[5] Tarrit, Katy \&Molleda, Julio \& Atkinson, Gary \& Smith, Melvyn \& C. Wright, Glynn \&Gaal, Peter. (2018). Vanishing point detection for visual surveillance systems in railway platform environments. Computers in Industry. 98. 153-164.

[6] Rivas, Alberto \&Chamoso, Pablo \& González Briones, Alfonso \&Corchado Rodríguez, Juan. (2018), "Detection of Cattle Using Drones and Convolutional Neural Networks," Sensors, 18.

[7] W. Xue, T. Jiang and J. Shi, "Animal intrusion detection based on convolutional neural network," 2017 17th International Symposium on Communications and Information Technologies (ISCIT), Cairns, QLD, 2017, pp. 1-5.

[8] SlavomirMatuska, Robert Hudec, PatrikKamencay, Miroslav Benco, Martina Zachariasova, Classification of Wild Animals based on SVM and Local Descriptors,AASRI Procedia, Volume 9, 2014, Pages 25 30, ISSN 2212-6716

[9] Alexander Gomez Villa, Augusto Salazar, Francisco Vargas,Towards automatic wild animal monitoring: Identification of animal species in camera-trap images using very deep convolutional neural networks, Ecological Informatics, Volume 41, 2017, Pages 24-32, ISSN 1574 9541.

[10] Z. Zhang, Z. He, G. Cao and W. Cao, "Animal Detection from Highly Cluttered Natural ScenesUsing Spatiotemporal Object Region Proposals and Patch Verification," in IEEE Transactions on Multimedia, vol. 18, no. 10, pp. 2079-2092, Oct. 2016.

[11] Z. Zhang, T. X. Han and Z. He, "Coupled ensemble graph cuts and object verification for animal segmentation from highly cluttered videos," 2015 IEEE International Conference on Image Processing (ICIP), Quebec City, QC, 2015, pp. 2830-2834.

[12] Verma G.K., Gupta P. (2018) Wild Animal Detection Using Deep Convolutional Neural Network. In: Chaudhuri B., Kankanhalli M., Raman B. (eds) Proceedings of 2nd International Conference on Computer Vision \& Image Processing. Advances in Intelligent Systems and Computing, vol 704. Springer, Singapore.

[13] Sharma, K.U. and Thakur, N.V., 2014. An Approach For Single Object Detection In Images. International Journal of Image Processing (IJIP), 8(5), p.278.

[14] Kumar, S., \& Singh, S.K. (2016). Monitoring of pet animal in smart cities using animal biometrics. Future Generation Comp. Syst., 83, 553-563.

[15] Sharma S and Shah D, 2013, A brief overview on different animal detection method, Signal and Image Processing: An International Journal, vol. 4, pp: 77-81.

[16] Verma G.K., Gupta P. (2018) Wild Animal Detection Using Deep Convolutional Neural Network. In: Chaudhuri B., Kankanhalli M., Raman B. (eds) Proceedings of 2nd International Conference on Computer Vision \& Image Processing. Advances in Intelligent Systems and Computing, vol 704. Springer, Singapore

[17] Trnovszky, Tibor \&Kamencay, Patrik\&Orješek, Richard \&Benco, Miroslav \&Sykora, Peter. (2017), Animal Recognition System Based on Convolutional Neural Network. Advances in Electrical and Electronic Engineering. 15. 10.15598/aeee.v15i3.2202.

[18] Ran, Y., Weiss, I., Zheng, Q. and Davis, L.S., 2007. Pedestrian detection via periodic motion analysis. International Journal of Computer Vision, 71(2), pp.143-160.

[19] S. U. Sharma and D. J. Shah, "A Practical Animal Detection and Collision Avoidance System Using Computer Vision Technique," in IEEE Access, vol. 5, pp. 347-358, 2017.

[20] Manjot Kaur and Rajneesh Randhawa, (2019) Animal Detection: Techniques, Challenges and Future Scope, International Journal of Innovative Technology and Exploring Engineering (IJITEE), vol.9.

[21] Sharma, S.U. and Shah, D., 2017. DESIGN AND DEVELOPMENT OF ANIMAL DETECTION ALGORITHM USING IMAGE PROCESSING.

[22] Neethirajan, S., 2017. Recent advances in wearable sensors for animal health management. Sensing and Bio-Sensing Research, 12, pp.15-29.

[23] Pathare, S.P., 2015. Detection of black-backed jackal in still images (Doctoral dissertation, Stellenbosch: Stellenbosch University). 
[24] Koik, B.T. and Ibrahim, H., 2012. A literature survey on animal detection methods in digital images. International Journal of Future Computer and Communication, 1(1), p.24.

[25] Figueroa, K., Camarena-Ibarrola, A., García, J. and Villela, H.T. 2014, November. Fast automatic detection of wildlife in images from trap cameras. In Iberoamerican Congress on Pattern Recognition (pp. 940-947). Springer, Cham.

[26] G. C. White and R. A. Garrott, Analysis of wildlife radio-tracking data. Elsevier, 2012.

[27] A. Swanson, M. Kosmala, C. Lintott, R. Simpson, A. Smith, and C. Packer, "Snapshot Serengeti, high-frequency annotated camera trap images of 40 mammalian species in an African savanna," Scientific Data, vol. 2, p. 150026, 2015.

[28] M. S. Bartlett, G. C. Littlewort, M. G. Frank, and K. Lee, "Automatic decoding of facial movements reveals deceptive pain expressions," Curr. Biol., vol. 24, no. 7, pp. 738-743, 2014

[29] Blumrosen, G., Hawellek, D. and Pesaran, B., 2017. Towards automated recognition of facial expressions in animal models. In Proceedings of the IEEE International Conference on Computer Vision (pp. 2810-2819).

[30] Cai, C. and Li, J., 2013, October. Cattle face recognition using local binary pattern descriptor. In 2013 Asia-Pacific Signal and Information Processing Association Annual Summit and Conference (pp. 1-4). IEEE.

[31] Koik, B.T. and Ibrahim, H., 2012. A literature survey on animal detection methods in digital images. International Journal of Future Computer and Communication, 1(1), p.24.

[32] Christiansen, P., Steen, K., Jørgensen, R. and Karstoft, H., 2014. Automated detection and recognition of wildlife using thermal cameras. Sensors, 14(8), pp.13778-13793.

[33] Nguyen, H., Maclagan, S.J., Nguyen, T.D., Nguyen, T., Flemons, P., Andrews, K., Ritchie, E.G. and Phung, D., 2017, October. Animal recognition and identification with deep convolutional neural networks for automated wildlife monitoring. In 2017 IEEE international conference on data science and advanced Analytics (DSAA) (pp. 40-49). IEEE.

[34] Psota, E.T., Mittek, M., Pérez, L.C., Schmidt, T. and Mote, B., 2019. Multi-Pig Part Detection and Association with a Fully-Convolutional Network. Sensors, 19(4), p.852.

[35] Jukan, A., Masip-Bruin, X. and Amla, N., 2017. Smart computing and sensing technologies for animal welfare: a systematic review. ACM Computing Surveys (CSUR), 50(1), p.10.

[36] Anu, V.M., Deepika, M.I. and Gladance, L.M., 2015, February. Animal identification and data management using RFID technology. In International Confernce on Innovation Information in Computing Technologies (pp. 1-6). IEEE.

[37] Zhu, C., Li, T.H. and Li, G., 2017. Towards automatic wild animal detection in low quality camera-trap images using two-channeled perceiving residual pyramid networks. In Proceedings of the IEEE International Conference on Computer Vision (pp. 2860-2864).

[38] Giraldo-Zuluaga, J.H., Salazar, A., Gomez, A. and Diaz-Pulido, A., 2017, November. Recognition of Mammal Genera on Camera-Trap Images Using Multi-layer Robust Principal Component Analysis and Mixture Neural Networks. In 2017 IEEE 29th International Conference on Tools with Artificial Intelligence (ICTAI) (pp. 53-60). IEEE. 